\title{
Unified Identification for Event Driven Service in User-Centric Environments
}

\author{
Soong-Hee Lee, Dong-Il Kim, Hyung-Goo Jeon, and Jong-Wook Jang, Member, KIMICS
}

\begin{abstract}
Considerations for user-centric environments are regarded as essential for the successful deployment of convergence services in BcN. Event driven service (EDS) is regarded as a typical convergence service converging different functions of multiple service providers to support user-centric environments. This paper first describes the deployment model and service scenario of EDS, a convergence service for user-centric environments in $\mathrm{BcN}$. The user-centric environments stimulates the unified identifier management, namely unified identification (U-ID), to enable users to be provided convergence services without awareness of multiple providers. Then the designed structure and implementation results are given.
\end{abstract}

Index Terms - U-ID for EDS, BcN convergence service

\section{INTRODUCTION}

CONVERGENCE services should operate seamlessly across $\mathrm{BcN}$ (or NGN) infrastructures that will be provided in the near future. Seamless interconnection between networks managed by different network providers and seamless interoperability of services offered by different application service providers (ASPs) are required[1]. $\mathrm{BcN}$ will stimulate an evolution to the multi-service and multinetwork provider environment or 'multi-provider' interoperable environment for user-centric services. Future networks such as $\mathrm{BcN}$ will support user-centric environments according to this trend.

The user-centric environments evidently demand management of multiple identifiers (IDs) as different networks or services have different types of identifiers for their profile managements. These multiple IDs used in the convergence services require an efficient method for manageable service provision that naturally leads to the introduction of a kind of unified ID management (U-ID) system.

There have been several efforts to deal with the issue of ID managements such as RFID, ENUM, and UCI, etc.[2 8]. Electronic or E.164 NUMber Mapping (ENUM) is defined by the Internet Engineering Task Force (IETF) in RFC 3761, that is the mapping of telephone numbers to uniform resource identifiers

\footnotetext{
Manuscript received February 10, 2011; revised March 11, 2011; accepted March 25, 2011.

This work was supported by Grant from Inje University, 2007.
}

(URIs)[4]. The purpose of ENUM is limited as to enable the convergence between the PSTN and the Internet only. IETF RFC 4179 deals with universal content identifier (UCI) about the standardized structure of identification codes for relation between various digital contents[5]. These standards or studies only cover simple matches between limited types of identifiers for limited areas for a single provider that does not consider interactions between multiple service providers. Single sign on (SSO), defined in Y.IDMsec[6] for ID management in NGN, could manage multiple IDs in a unified way using unified login, but presents a limited feature for service provision while we must consider a unified ID management for convergence services in the wider range.

$\mathrm{BcN}$ is the future network that is expected to provide convergence services that will play main roles in the future user-centric environments. One of such services is the context aware service. Since the concept of "context awareness" was introduced by Schilit[9] as "devices may have information about the circumstances under which they are able to operate and based on rules, or an intelligent stimulus, react accordingly", there have been much interests on the issue of context awareness or context aware service, especially in the ubiquitous computing filed. However, they are mainly related with web-based applications or mobile communications[9 11]. There had been no efforts to deploy the context aware service as one of the NGN or $\mathrm{BcN}$ convergence services before event driven service (EDS) was introduced in Y.Sup3[1] though $\mathrm{BcN}$ is regarded as the main infrastructure for the future ubiquitous environment.

These trends force us to explore a new paradigm of a $\mathrm{BcN}$ convergence service considering the unified ID management and context aware service paradigm together, i.e., U-ID for EDS. We first outlined the service feature using scenario-based approach, designed the structure and procedure, and implemented. The implemented result requires verification process in the actual environment of $\mathrm{BcN}$.

Convergence Service will emerge as an important business model in the NGN environments, e.g., $\mathrm{BcN}$ in Korea. Hence, much effort needs to be concentrated into establishing the new concepts of convergence service scenarios via research and standardization activities. Moreover, we need to explore technical basis on unified management of identification, certification, and profile management to provide the convergence services. 
Therefore, the testing and verification process is required for confirmation after applying the developed results into the actual network. This leads to the verification tests using the network, that is similar with the future networks, and applying the convergence service scenario, that is expected to be applied into the future networks. Considering Korea Advanced Research Network (KOREN) for this purpose of verification tests is very important in that KOREN is the very infrastructure for testing $\mathrm{BcN}$, that will be deployed in the near future, and the most appropriate testing environment consequently.

This paper first describes the service scenario of U-ID for EDS, a convergence service in $\mathrm{BcN}$. Its structure and required procedures are described next. The applied scheme and the results after implementation are explained and the conclusion is lastly given.

\section{SERVICE SCENARIO OF A BCN CONVERGENCE SERVICE, U-ID FOR EDS}

Event driven service (EDS) is listed as a typical convergence service performing convergence between functions owned by multiple service providers[1]. EDS provides users with the relevant information and the communication by the various personal conditional environments. An alternative terminology for events for network-related applications is 'triggers' - these are assumed to be included in the present description.

Events could be defined as any activities which occur within a service area caused by user actions, connectivity signals, signals related to service selection and service availability, signals classifying provider and associated control signals, and billing signals.

Events can be managed, for example, as part of a context-aware application. EDS could carry out a procedure for processing user's requested information about mobile network-based entities, such as the combination with various applications, such as a location based service (LBS) and area information. In $\mathrm{BcN}$ environments, the user can be serviced his/her customized EDS in the network. The EDS automatically connects the appropriate CTE consistent with a user profile. EDS can also send the collected information to other requesting users by way of event information and using the procedures of combination with other services.

The user can record/retrieve the user-related information to/from the information base through EDS. The information base may initially reside in the server of the provider side. The cache of the CTE may partly store the information and can be autonomously updated.

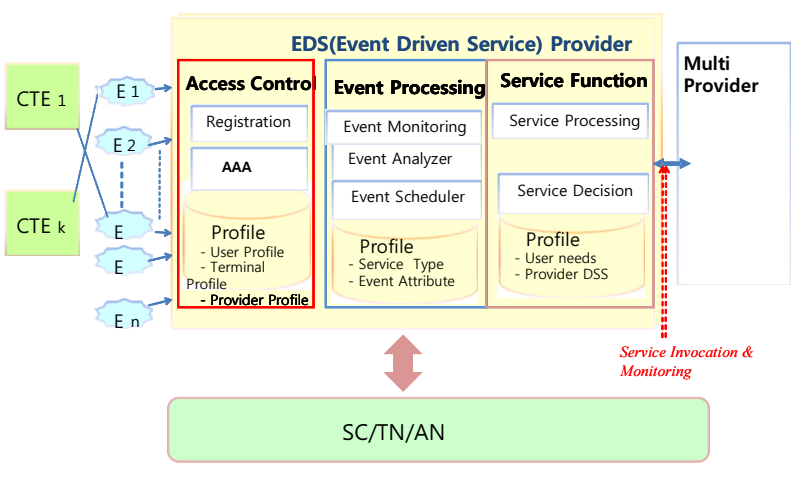

Fig. 1. Service model of EDS.

EDS consists of Access Control Function, Event Processing Function, and Service Function as shown in Fig. 1[1]. The services to be converged include LBS (Location Based Service), DSS (Discovery Service), and Push service, etc. in the multi-provider environment. $\mathrm{E}_{1}$, $E_{2, \ldots}, E_{n}$ refer to events or triggers.

Access Control function covers registrations of user profiles, terminal profiles, and provider profiles for providing the proper application services with applying AAA to users. The user requesting services could be offered with performing AAA function on the basis of preregistered profile data such as user ID, and Terminal ID, etc. These multiple IDs used in the profiles demand an efficient method for manageable service provision that naturally leads to the introduction of a unified ID management system in the next chapter.

\section{DESIGNED STRUCTURE FOR U-ID FOR EDS}

Event Processing function makes the choice of the best suited access option, by taking into consideration the application requirements and user preferences by analyzing event attributes. All this information is maintained and structured in profiles, which are located in the Access Profile of the Access Control. These profiles can be accessed by the service function in order to combine link-specific parameters with user preferences and application requirements.

EDS can provide the more valuable services to users by efficiently managing the resources or functions residing in the multiple providers (ASPs or carriers). One of the methods required to achieve this goal is the unified ID management system, called U-ID in this paper. Event Driven Service (EDS), combined with Unified ID management (U-ID), i.e., U-ID for EDS will be described next.

U-ID(Ubiquitous Identifier) can function as a convergence service administrator for offering convergence services for user-centric environments. Fig. 2 is demonstrating the concept of U-ID for user-centricity. 


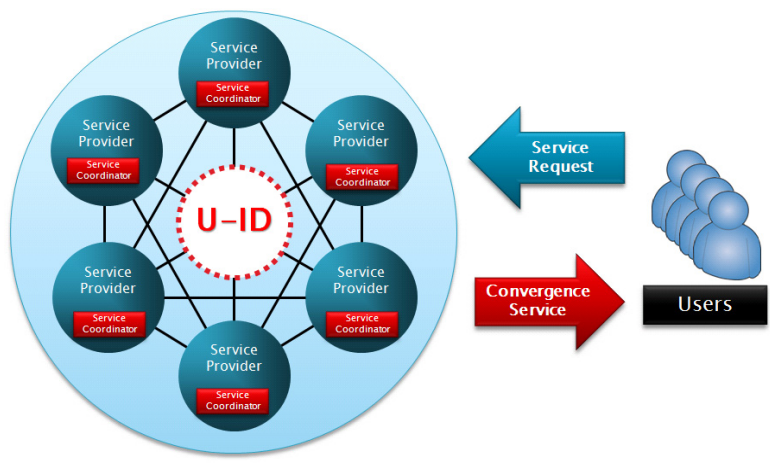

Fig. 2. The concept of U-ID for user-centricity.

As shown in Fig. 2, U-ID carries out ID management including unified authentication, unified accounting, and unified profile management between service providers.

U-ID needs to communicate with EDS functions and unification profiles for the required service functions. UID can be applied to the profile-related procedures for the EDS provision.

EDS requires technologies that senses and analyzes events that happen on the real time basis, which provides services as the responses to the analyzed events. With the aid of U-ID, EDS enables convergence services between various networks and providers, which provide the customized services to the users and the enterprise environments on the real time basis, etc. This type of service proivision is called U-ID for EDS in this paper. Fig. 3 shows the interactions required for U-ID for EDS.

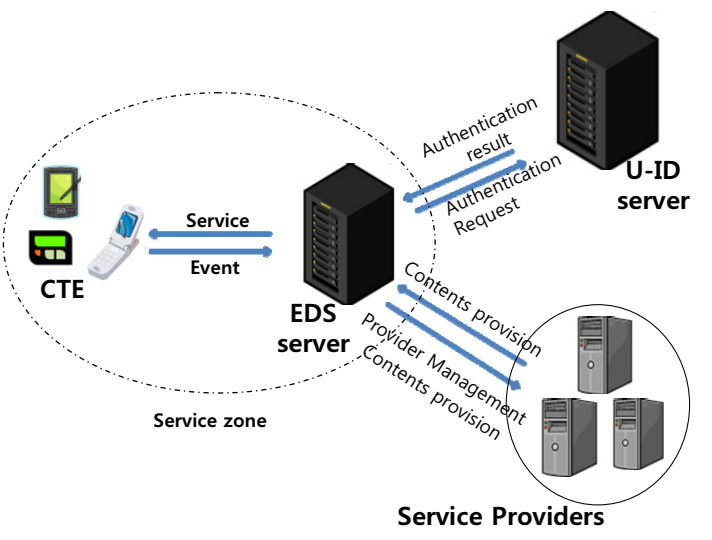

Fig. 3. Interactions for U-ID for EDS.

EDS is provided combining the service functions listed in Figure 1, i.e., access control, event processing, service function. The detailed procedures in these functions are described in the next clauses.

The first function to be considered is Access Control function. This function requests the authentication for the access within the service area and manages the attached terminals. If attached terminals enter the service area without the users' requests, they will be automatically managed using the access codes in the terminals. Access Control includes the following interactions:
(1) Registration: Terminals or users that entered service area are registered into the Access Control system. This process is carried out without access requests for immediate reaction to the possible occurrence of events

(2) Authentication, authorization: The appropriate rights are authorized according to the information that passed U-ID authentication process. The personal information that passed U-ID authentication is analyzed and used to authorize the range of service rights that fits to the service user level and system capacity.

(3) Profiles

- User Profile that passed U-ID authentication is registered to enable the efficient user management and service.

- Terminal Profile registered to the current service area is registered to enable the analysis of available services. For example, a terminal that supports not DMB but WLAN will broadcast the intended contents via IP streaming service.

In Event Processing function, all events occurring within service area are managed and controlled applying this function. Event processing function performs eventrelated procedures as follows:

(1) Event Monitoring: User/terminal-requested Events are monitored and delivered to the manager on the realtime basis.

(2) Event Analysis: Events are analyzed to investigate the current status or trigger service providing. Events caused by users are statistically analyzed and reported to the manager that will appropriately cope with the upcoming events using the analyzed result.

(3) Event Scheduler: Events are scheduled to be provided to users for more efficient processing. Events caused by users are scheduled to enable users to efficiently manage their own events.

(4) Profiles: There are two types of event attributes to be stored in the profile, one for service and another for billing as follows:

- TYPE I (Service) profile stores the service features provided to the users;

- TYPE II (Billing) profile stores the billing information for services provided to the users.

Service Function provides more efficient services to users after user and terminal profiles are analyzed. Service providers are also managed. Service function includes the following procedures:

(1) Service Processing: Users and providers are linked and managed to prevent duplicate or incorrect service providing with this function. Providers for the same service or user-requested service are managed to guarantee service reliability to the users. In addition, services that were already in provision are analyzed and services are provided to enable users to actively interact (e.g., Push service). 
(2) Service Decision: Services requested by users are linked with providers which can provide the required service. The priority or popularity can be used to choose the most appropriate service providers.

(3) Profiles: There are profiles for user need and provider DSS as follows:

- User Need profile stores services selected by users or provided to users that are used for services such as Push service;

- Provider DSS profile manages a kind of directory in which service provider registered.

With Service Control function, the services are controlled during the service provision. The efficient and easy management of QoS is possible by managing the provided services, terminals, and networks. It is especially needed for the case that multiple users demand the same service or that a user is provided services simultaneously.

By Service Invocation \& Monitoring function, services selected and requested by users are invocated and monitored.

\section{IMPLEMENTATION OF U-ID FOR EDS}

The U-ID for EDS is designed on the structure and procedure level applying the service scenario described in the last chapter. The details of the designed structure and procedures are explained in this chapter.

U-ID for EDS is designed to consist of two parts, U-ID system and EDS system. We designed each system and considered interaction between two systems. The designed U-ID system roughly consists of two parts, a UID manager and the unification profile. Fig. 4 and 5 show the structure of the U-ID manager, and the structure of the unification profile each.

As shown in Fig. 4, the U-ID manager is composed of eight functional blocks, i.e., Identifier Functions, Authentication Functions, Accounting Process, Administrator Management, Unification Profile Management, User Communication Management, Service Provider Communication Functions, and Communication \& Data Confidentiality. The unification profile includes seven profiles and one function as shown in Fig. 5.

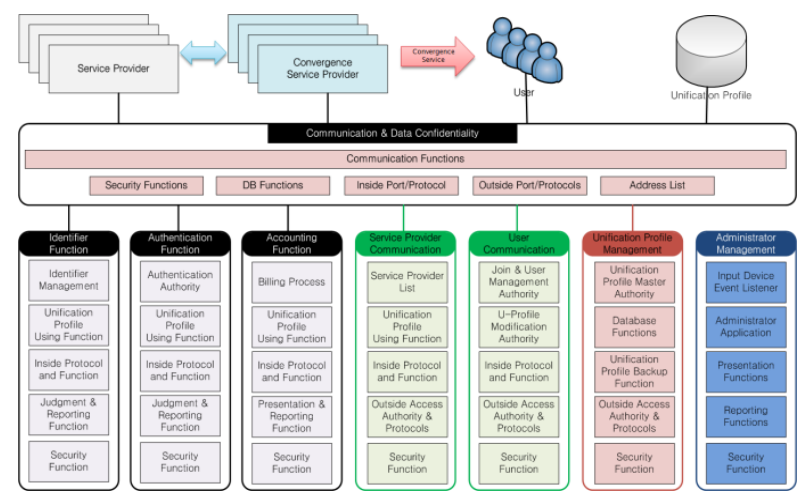

Fig. 4. Structure of the U-ID manager.

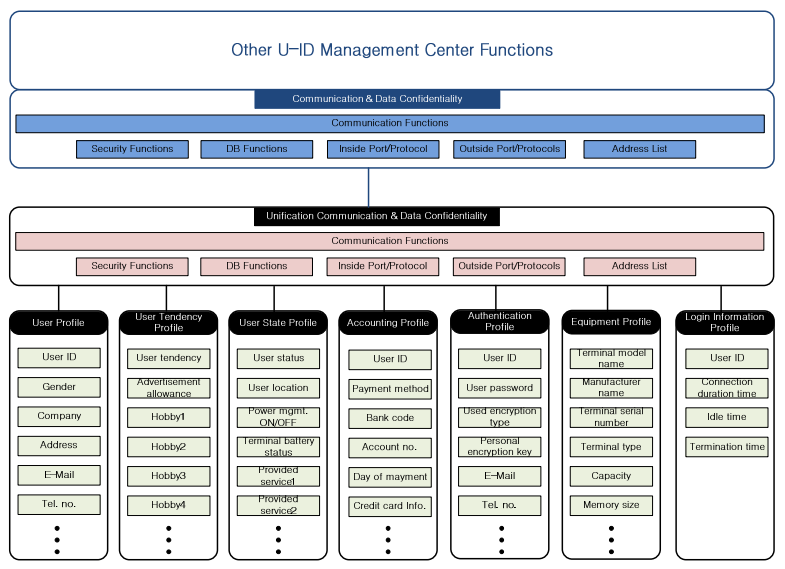

Fig. 5. Structure of the unified profile.

The designed structure needs to be verified through implementation and actual deployment. One of the practical ways to achieve this goal is to implement the service systems according to the designed structure as brief as possible and applies to the network that is possibly closest to the future network environments. We implement the U-ID for EDS system applying the architecture of the implemented software shown in Fig. 6. The implemented software consists of the EDS system and the U-ID system communicating each other for exchange of identification and billing data.

Based on the architecture in Fig. 6, the structure of the profile and communication protocol is designed and the software for U-ID for EDS is to be implemented using SQL Database, Visual C++, etc. Details of U-ID for EDS procedures needed for implementation are described next.

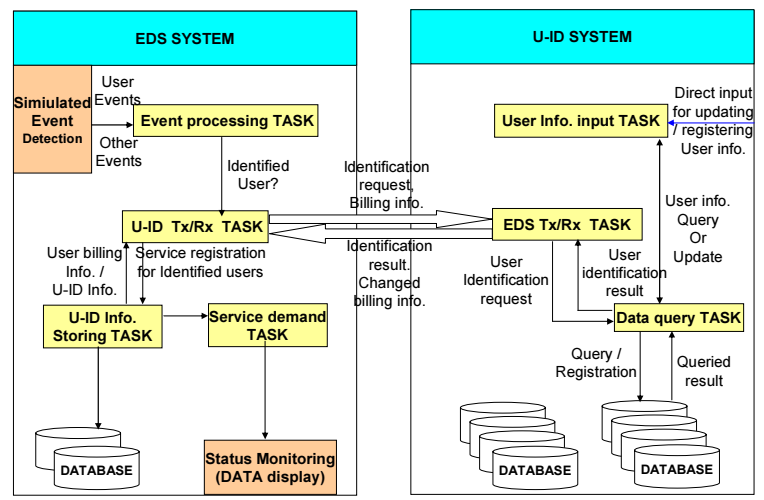

Fig. 6. Architecture of the implemented software for U-ID for EDS.

First, event detection and service information output must be considered. It is required to detect events from the user side, demands authentication, and output service information about the authenticated user. 
Storing, lookup, and modification of EDS server information also need to be considered. It is required to store, lookup, and modify information from/to EDS server.

We need to access to U-ID server. It is required to access U-ID server if needed, especially when the user authentication is required or requested. $\mathrm{D} / \mathrm{B}$ information for U-ID is updated in this function.

Storing, lookup, and modification of U-ID server information are left to be solved. It is required to store, lookup, and modify information from/to U-ID server for the purpose of registration of new users or terminals, authentication and authorization for the registered users or terminals, monitoring, etc.

The last issue is users' access to EDS server. It is required to access EDS server, when the user authentication is required or requested. Registration for new users and D/B for EDS update are also carried out.

Applying these considerations, the software for managing U-ID for EDS is implemented using SQL Database, Visual $\mathrm{C}++$, etc. The partial source code of the main task is shown in Fig. 7.

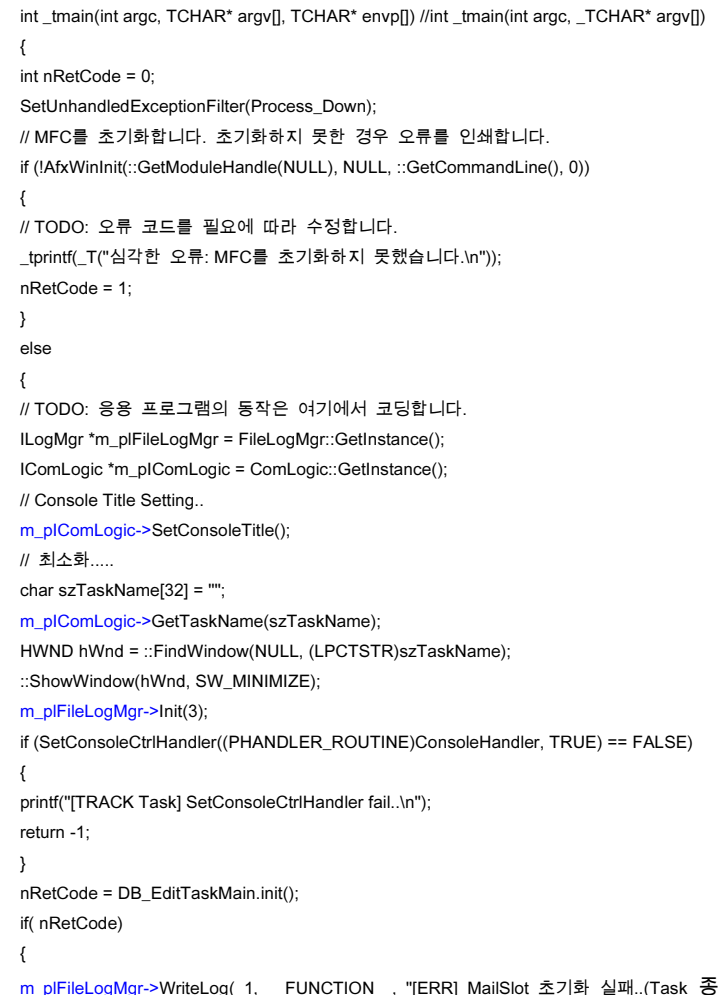

Fig. 7 A partial source code of the main task for U-ID for EDS.

The testing and verification process is required for confirmation before applying the developed results into the actual network. This leads to the verification tests using the network, that is similar with the future networks, and applying the convergence service scenario, that is expected to be applied into the future networks. Adapting and applying Korea Advanced Research Network (KOREN) for this purpose of verification tests is very important in that KOREN is the very infrastructure for testing $\mathrm{BcN}$, that will be deployed in the future, and the most appropriate testing environment consequently. The implemented result was verified through interworking tests through KOREN. The test was successful though the test was carried out just at the initial phase and this shows the possibilities of the designed structure as the service platform for providing convergence services for the usercentric environments

\section{CONCLUSION}

Researches on U-ID for EDS, and implementation and testing via KOREN were carried out. More specifically, architecture and roles, information flows, unified profiles were studied, designed and implemented to be tested in KOREN environment. The studied results were presented as the contributions in the international standardization meetings such as ITU-T.

The proposed scenario includes the functional architectures and main information flows for U-ID for EDS. Management software and unified profiles were established for interworking tests of U-ID for EDS in the KOREN environment. KOREN was used as a substitute for $\mathrm{BcN}$ in the test. The result of the test shows the possibilities of $\mathrm{BcN}$ (or KOREN) as the service platform for providing convergence services to the users in the user-centric environments, that will build infinite business chances in the future market of user-centric services. The detailed procedures need to be more studied for improvements and scalability.

\section{REFERENCES}

[1] ITU-T Y.Sup.3, "ITU-T Y.2000 series - Supplement on service scenarios for convergence services in a multiple network and application service provider environment", ITU-T ITU-T Y.2000 series Y.Sup.3, ITU-T, 2007.

[2] Sang-chul Shin, et. al., "Strategy for international standardization and wide spread of RFID/USN", KICS Journal, KICS, 2004.

[3] Young-gil Kim, "Data transmission in u-ID sensor network configuration using Bluetooth", ICKIMICS 2004 Proceedings, KIMICS, 2004.

[4] IETF RFC 3761, "The E.164 to Uniform Resource Identifiers (URI) Dynamic Delegation Discovery System (DDDS) Application (ENUM)", IETF RFC 3761, IETF, 2004.

[5] IETF RFC 4179, "Using Universal Content Identifier (UCI) as Uniform Resource Names (URN)", IETF RFC 4179, IETF, 2005.

[6] ITU-T Y.IdMsec, "NGN IDENTITY MANAGEMENT SECURITY", ITU-T Y.IdMsec, ITU-T, 2007.

[7] Spyridon Vassilaras et. al., "Architecture Framework for Device Single Sign On in Personal Area Networks", Lecture Notes in Computer Science, Springer, 2006.

[8] Tae-shik Sohn, et. al., "Single Sign-On System enabling Mutual Authentication in Multi Domain Environments", KISS Journal, 2001.

[9] B. Schilit, N. Adams, and R. Want., "Context-aware computing applications", IEEE Workshop on Mobile Computing Systems and 
Applications (WMCSA'94), Santa Cruz, CA, US, pp. 89-101, 1994.

[10] Rosemann, M., and Recker, J., Context-aware process design: Exploring the extrinsic drivers for process flexibility", 18th international conference on advanced information systems engineering proceedings of workshops and doctoral consortium, Namur University Press, Luxembourg, pp. 149-158, 2006.

[11] Soraya Kouadri Most'efaoui, B'eat Hirsbrunner, "Context Aware Service Provision", Proceedings of the IEEE/ACS International Conference on Pervasive Services (ICPS'04), IEEE Computer Society, 2004.

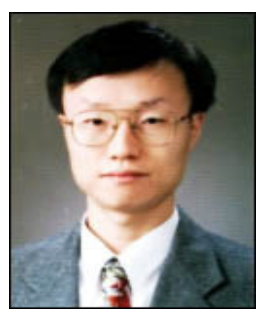

Soong-Hee Lee Received the B.S., M.S., and Ph.D. degrees from Kyungpook National University, Daegu in 1987, 1990, and 1995, respectively. From 1987 to 1977 , he was a member of research staff in Electronics and Telecommunications Research Institute. Since 1997, he has been with the Dep't of Information and Communications Engineering, Inje University as an associate professor. His research activities are in the area of next generation network technologies and services. He was designated as an IT Standard Expert on behalf of Korea by Ministry of Information and Communications in 2001. He is an Editor in the ITU-T SG13 and a Member of KIMICS.

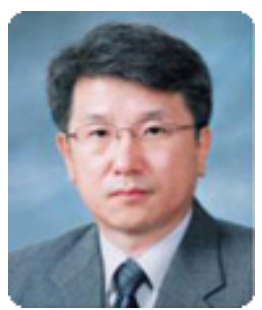

Dong-Il Kim Received the Ph.D degree in electronics information and communication engineering from Kwang-woon University, Korea, in 1992. Since 1991, he has been in Dong-eui University, Korea, where he is a professor at the information and communication engineering department. From 1983 to 1991 , he was a general manager at switching research center in LG information and communication institute. He was a visiting researcher at standardization research center, ETRI(Electronics and Telecommunications Research Institute, Korea), from 1998 to 1999 and also a chief of computer center at DEU, from 2003 to 2007. He is currently a director of KIMICS, member of international journal committee in KICS(Korea Institute of Communication Sciences), and international expert, the field of IT, in MIC(Ministry of Information and communications, Korea). His research interests are analysis of performance in a communication networks, protocols in wireless networks and standardization of IT.

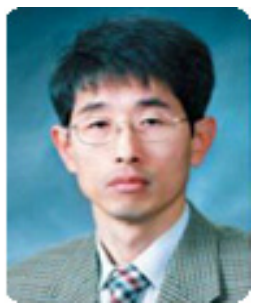

Hyung-Goo Jeon Was born in Jeon-Joo Korea on Dec. 1961. He received Bachelor degree at the department of electronics in Inha University in 1987, Master degree and Ph.D. degree from Yonsei University in Seoul Korea in 1992 and 2000 , respectively. He is now the associate professor of the information and communication engineering department in Dong-eui University. His research interests include digital communication, MIMO-OFDM,

WLAN, and IMT-2000.

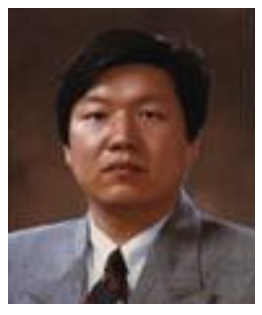

Jong-Wook Jang Received Ph.D. degree in computer engineering at Pusan National University in 1995. From 1987 to 1995 , he worked for ETRI as a senior member of research staff. He completed postdoctoral research in UMKC in 2000. Since 1995, he has been a professor at the computer engineering department of Dong-eui University. His research interests include vehicle networks, convergence networks, etc. 